\title{
Large-scale production of foot-and-mouth disease virus (serotype Asia1) VLP vaccine in Escherichia coli and protection potency evaluation in cattle
}

Yan Xiao ${ }^{1 \dagger}$, Hong-Ying Chen ${ }^{3 \dagger}$, Yuzhou Wang ${ }^{1}$, Bo Yin ${ }^{3}$, Chaochao Lv', Xiaobing Mo ${ }^{3,4}$, He Yan', Yajie Xuan ${ }^{1}$, Yuxin Huang ${ }^{1}$, Wenqiang Pang ${ }^{1}$, Xiangdong $\mathrm{Li}^{1}$, Y. Adam Yuan ${ }^{3,4^{*}}$ and Kegong Tian ${ }^{1,2^{*}}$

\begin{abstract}
Background: Foot-and-mouth disease (FMD) is an acute, highly contagious disease that infects cloven-hoofed animals. Vaccination is an effective means of preventing and controlling FMD. Compared to conventional inactivated FMDV vaccines, the format of FMDV virus-like particles (VLPS) as a non-replicating particulate vaccine candidate is a promising alternative.

Results: In this study, we have developed a co-expression system in E. coli, which drove the expression of FMDV capsid proteins (VPO, VP1, and VP3) in tandem by a single plasmid. The co-expressed FMDV capsid proteins (VPO, VP1, and VP3) were produced in large scale by fermentation at $10 \mathrm{~L}$ scale and the chromatographic purified capsid proteins were auto-assembled as VLPs in vitro. Cattle vaccinated with a single dose of the subunit vaccine, comprising in vitro assembled FMDV VLP and adjuvant, developed FMDV-specific antibody response (ELISA antibodies and neutralizing antibodies) with the persistent period of 6 months. Moreover, cattle vaccinated with the subunit vaccine showed the high protection potency with the $50 \%$ bovine protective dose $\left(\mathrm{PD}_{50}\right)$ reaching $11.75 \mathrm{PD}_{50}$ per dose.

Conclusions: Our data strongly suggest that in vitro assembled recombinant FMDV VLPs produced from E. coli could function as a potent FMDV vaccine candidate against FMDV Asia1 infection. Furthermore, the robust protein expression and purification approaches described here could lead to the development of industrial level large-scale production of E. coli-based VLPS against FMDV infections with different serotypes.
\end{abstract}

Keywords: Foot-and-mouth disease, Serotype Asia 1, Virus-like particle vaccine, Fifty percent protection dose $\left(\mathrm{PD}_{50}\right)$, Large scale production in E. coli

\section{Highlights}

1. A co-expression system in $E$. coli driving the expression of FMDV capsid proteins (VP0, VP1, and VP3) in tandem by a single plasmid is constructed.

2. Large-scale chromatographic purified FMDV capsid proteins are auto-assembled into VLPs in vitro.

\footnotetext{
* Correspondence: dbsyya@nusri.cn; tiankg@263.net

${ }^{\dagger}$ Equal contributors

${ }^{3}$ National University of Singapore (Suzhou) Research Institute, 377 Lin Quan Street, Suzhou Industrial Park, Jiangsu 215123, China

${ }^{1}$ National Research Center for Veterinary Medicine, Road Cuiwei, High-Tech

District, Luoyang 471003, People's Republic of China

Full list of author information is available at the end of the article

3. VLP-based FMDV vaccine induces strong and persistent humoral immunity with the period of 6 months

4. VLP-based FMDV vaccine displays strong protective efficacy against FMDV challenge

\section{Background}

Foot-and-mouth disease (FMD) is a highly contagious and devastating disease of cloven-hoofed animals that causes significant economic losses worldwide [1]. FMD is endemic in many countries including parts of Asia, Africa, South America, and at the periphery of the European Union [2]. The causative agent, foot-and-mouth 
disease virus (FMDV), belongs to the genus Aphthovirus of the family Picornaviridae. The virus consists of seven serotypes, including type A, O, C, Asia 1, and South African Territories (SAT) 1-3 [3].

Conventional inactivated whole-virus vaccines are the primary measure for controlling the disease in most endemic areas [3]. Such vaccines have played important roles in eliminating the disease from some areas of the world [4]. However, potential dangers still accompany the use of whole virus vaccines because of the incomplete inactivation of FMDV in large-scale production and the risk of escape of the live virus from vaccine producing facilities [5]. Thus, a number of different types of vaccines have been developed including synthesized peptide vaccines [6], recombinant virus-vectored vaccines [7], virus-like particles (VLPs) subunit vaccines [8-10], and DNA vaccines $[11,12]$. Among these, the VLP subunit vaccine that contains all of the immunogenic sites present on the intact virions but lacks nucleic acid component is safe and effective. Development of VLP vaccines has been attempted by using various expression platforms including eukaryote and prokaryote expression systems $[8,13]$. Compared to the eukaryote expression system, the production of recombinant proteins in the prokaryote system, such as $E$. coli, is considered to be less laborious and less expensive.

The FMDV genome contains a single large open reading frame. The capsid of FMDV consists of three structural proteins VP0, VP1 and VP3, which are the cleavage products of the $\mathrm{P} 1-2 \mathrm{~A}$ capsid precursor polypeptide yielded from viral $3 \mathrm{C}$ protease digestion. During virus maturation, capsid protein VPO is further cleaved to make capsid proteins VP2 and VP4. Either capsid proteins VP0, VP1 and VP3 or capsid proteins VP1, VP2, VP3 and VP4 are able to form the icosahedral capsid particles during the different stages of virus maturation by self-assembly [8]. In addition, two research groups successfully produced empty icosahedral capsid particles by expression of VP0, VP1 and VP3 capsid proteins in $E$. coli by fusing these proteins with small ubiquitin-like modifier (SUMO) tag [8, 14]. Furthermore, Guo et al. [8] evaluated the immunogenicity of the in vitro assembled VLPs in cattle, which is composed of capsid proteins VP0, VP1 and VP3. However, both published methods used 2 and 3 plasmids with different antibiotic selection markers, respectively, to successfully co-express the FMDV capsid protein in soluble form. However, the multiple antibiotic selection pressure by using multiple vectors with different antibiotic selection markers could significantly reduce the bacterial growth and could raise environmental and food security concerns. Moreover, the VLP preparation protocols provided by these two papers may not be suitable for large scale production of VLP vaccine at industrial level since neither analytical size exclusion chromatographic approach nor sucrose gradient ultracentrifugation approach was considered as industrially favorable one for vaccine preparation. Furthermore, the reported yield of VP0-VP1-VP3 ternary complexes was $\sim 5 \mathrm{mg} / \mathrm{L}$ by using two plasmids and even less yield of ternary complexes was achieved by using three plasmids $[8,14]$.

In this study, we optimized the E. coli co-expression system by co-expressing SUMO fused full-length FMDV capsid proteins (VP0, VP1, and VP3) in tandem driven by a single plasmid and selected by a single antibiotic. In our case, the co-expressed FMDV capsid proteins (VP0, VP1, and VP3) were produced by fermentation at $10 \mathrm{~L}$ scale with the protein level reaching $\sim 50 \mathrm{mg} / \mathrm{L}$ culture without further optimization. Furthermore, we optimized the purification protocols by obtaining $\sim 90 \%$ pure FMDV capsid proteins without size exclusion chromatographic purification, which is not preferred at industrial level due to the cost. The expressed full-length capsid proteins VP0, VP1, and VP3 were in vitro assembled into VLPs, which showed high protection potency with $11.75 \mathrm{PD}_{50}$ per dose when applied as subunit vaccines in cattle. Taken together, our data provided a robust protocol, for the first time, leading to large-scale production of potent FMDV VLP vaccines against FMDV Asia1 infection.

\section{Methods}

\section{Production of recombinant protein and characterization of VLPs}

The full-length FMDV VP0, VP1 and VP3 genes were synthesized (Genewiz) and cloned into the plasmid pETSUMO, designated as pETSUMO-VP0, pETSUMO-VP1, and pETSUMO-VP3, respectively. Subsequently, the respective DNA fragments of the clones including the ribosome binding site, the SUMO and the FMDV VP gene, designated as RBS-SUMO-VP3, RBS-SUMO-VP1, and RBS-SUMO-VP0, were amplified by PCR from pETSUMO-VP3, pETSUMO-VP1 and pETSUMO-VP0, respectively. Then these DNA fragments were cloned into a single pET28b vector (Novagen, USA) in order. The recombinant plasmid obtained was designated as pET-TRI-Asia1-VP310. All the restriction enzymes were purchased from New England Labs and the polymerases were purchased from Qiagen.

The recombinant plasmid pET-TRI-Asia1-VP310 was transformed into E. coli BL21 (DE3) competent cells (Stratagene, USA) according to the manufacturer's manual. A single colony of transformant was grown in LuriaBertani (LB) medium containing $50 \mu \mathrm{g} / \mathrm{ml}$ kanamycin at $37{ }^{\circ} \mathrm{C}$ until the $\mathrm{OD}_{600}$ reached 0.8 . Then isopropyl $\beta$ D-thiogalactopyranoside (IPTG) was added to a final concentration of $0.4 \mathrm{mM}$. The culture was incubated for $4 \mathrm{~h}$ at $28{ }^{\circ} \mathrm{C}$ before subjected to sodium dodecyl sulfate polyacrylamide gel electrophoresis (SDS-PAGE) 
and western-blot to confirm the expression of recombinant protein.

Next, the fermentation was performed in $10 \mathrm{~L}$ bioreactor (Baoxing, Shanghai, China). The media for the primary seed, secondary seed, and fermentation of the cultures were $\mathrm{LB}$ comprising tryptone $10 \mathrm{~g} / \mathrm{L}$, yeast extract $5.0 \mathrm{~g} / \mathrm{L}$, $\mathrm{NaCl} 10 \mathrm{~g} / \mathrm{L}$, and kanamycin $50 \mathrm{~g} / \mathrm{L}$. The primary seed culture was prepared by the transfering of $1 \mathrm{~mL}$ of glycerol stock of recombinant $E$. coli strain to $50 \mathrm{~mL}$ of $\mathrm{LB}$ medium in a $250-\mathrm{mL}$ flask, which was then aerobically incubated overnight at $37{ }^{\circ} \mathrm{C}$. The secondary seed culture was prepared by inoculating $2 \mathrm{~L}$ flasks containing $500 \mathrm{~mL}$ of LB medium with $5 \mathrm{~mL}$ of primary seed culture and cultivating overnight at $37{ }^{\circ} \mathrm{C}$ and $220 \mathrm{rpm}$. Flask of the secondary seed culture were then inoculated into $5.0 \mathrm{~L}$ of medium in a $10-\mathrm{L}$ bioreactor and cultivating at $37^{\circ} \mathrm{C}$ and $\mathrm{pH}$ 7.0, in which dissolved oxygen was maintained above $20 \%$. The fed-batch process of recombinant protein production in 10-L bioreactor was divided into three phases. Phase I was an aerobic batch cultivation at $37^{\circ} \mathrm{C}$ for $2 \mathrm{~h}$. Phase II was a fed-batch cultivation at $37{ }^{\circ} \mathrm{C}$ for $4 \mathrm{~h}$ in which $300 \mathrm{~g} / \mathrm{L}$ concentrated glucose was fed continuously to maintain the required specific growth rate of bacteria. In Phase III was an induction process at $37{ }^{\circ} \mathrm{C}$ for $6 \mathrm{~h}$ using isopropyl- $\beta$-d-thiogalactoside (IPTG) at a final concentration of $0.4 \mathrm{mM}$ to induce the expression of recombinant protein. The bacteria were harvested by centrifugation and the cell pellets were re-suspended at the buffer A (300 mM NaCl, $20 \mathrm{mM}$ Tris- $\mathrm{HCl}$ [pH7.0]). The cells were disrupted by homogenizer (Avestin) and the cell supernatant was run through the His-affinity chromatographic purification, washed by buffer B $(50 \mathrm{mM}$ Tris- $\mathrm{HCl}, 0.3 \mathrm{M} \mathrm{NaCl}, 0.2 \mathrm{mM}$ EGTA, and $40 \mathrm{mM}$ imidazole) and eluted by using $500 \mathrm{mM}$ imidazole. The SUMO protein tag was removed by Ulp1 digestion, followed by Q Sepharose Fast Flow anion exchange chromatographic purification (GE Healthcare). The purified proteins were concentrated by using Amicon centrifuge tubes (Millipore) for small scale preparation or by using Centramate TFF cassette (PALL) for large scale production. The purified recombinant FMDV VP3, VP1 and VP0 proteins were resuspended with $50 \mathrm{mM}$ Tris- $\mathrm{HCl}$, supplemented by a series of $\mathrm{NaCl}$ with different concentrations. The purified recombinant FMDV VP3, VP1 and VP0 proteins have been almost completely re-assembled into VLPs in a buffer of $50 \mathrm{mM}$ Tris- $\mathrm{HCl}$ and $500 \mathrm{mM} \mathrm{NaCl}$.

\section{Transmission electron microscope}

Purified VLPs were adsorbed onto a copper grid for $5 \mathrm{~min}$ at room temperature. The grids were dried gently using filter paper and stained with $3 \%$ of phosphotungstic acid (PTA) for $5 \mathrm{~min}$. The excess liquid was removed with filter, and the samples were examined under a TEM at $80 \mathrm{kV}$ (FEI).

\section{Dynamic Light Scattering (DLS) analysis of FMDV capsid VLPs}

Dynamic light scattering studies were carried out on a DynaPro Light Scattering instrument (Wyatt Technology Europe $\mathrm{GmbH}$, Dernbach, Germany) with protein concentrations at A280 nm of 1.0, in buffer containing $50 \mathrm{~mm}$ sodium phosphate, $\mathrm{pH} 6.5$, and $5 \mathrm{~mm}$ DTT.

\section{Vaccine preparation}

The purified capsid protein and adjuvant ISA 206 (SEPPIC, France) was emulsified using a RW 20 homogenizer (IKA, German) under sterile conditions at $300 \mathrm{rpm}$ for $5 \mathrm{~min}$, in a ratio of 46:54 (antigen:adjuvant) in volume. The preparations were stored at $4{ }^{\circ} \mathrm{C}$ until use.

\section{Animals and experimental design \\ Vaccination and antibody monitor in cattle}

Ten six-month-old cattle, sero-negative of FMDV (LPBE antibody titers $<1: 16)$ were randomly assigned to two groups with 5 animals in each group. Group A and group B were vaccinated intramuscularly once with $2 \mathrm{ml}$ of FMDV Asia1 subunit vaccine containing $200 \mu \mathrm{g}$ and $100 \mu \mathrm{g}$ VLPs per cattle, respectively. Serum samples were collected at $21,60,90,120,150$ and $180 \mathrm{dpv}$, respectively.

\section{Fifty percent protection dose $\left(P D_{50}\right)$ test}

Seventeen six-month-old cattle, sero-negative of FMDV (LBPE antibody titers $\leq 1: 8$ ), were divided into four groups, and all animals were housed in an animal biosafety level 3 (BSL3) facility. Groups 1, 2 and 3 with 5 animals in each group were vaccinated intramuscularly one dose subunit vaccine, $1 / 3$ dose subunit vaccine, and $1 / 9$ dose subunit vaccine, respectively; Group 4 with 2 animals, sham-vaccinated with PBS, was used as challenge control.

$\mathrm{PD}_{50}$ test was performed as described by the OIE to test the potency of FMDV VLPs produced from E. coli as a vaccine candidate. All cattle were challenged by tongue intradermal inoculation with 10,000 bovine infective dose $50 \%\left(\mathrm{ID}_{50}\right)$ of FMDV strain Asial/JSL/ GSZY/06 per head at 28 days post vaccination (dpv). The animals were daily examined for possible occurrence of lesions in the mouth and feet. Any lesion at a site other than the inoculation site within 10 days postchallenge was considered as a rupture of immunity and considered as no protection.

Serum samples were collected at $0,7,14,21$, and $28 \mathrm{dpv}$. The bovine $\mathrm{PD}_{50}$ content of the vaccine was calculated based on the Reed-Muench method from each animal protected in each group. The animal trial in this study was approved by the Animal Care and Ethics Committee of China National Research Center for Veterinary Medicine 
with reference number 2015062503, and conventional animal welfare regulations and standards were taken into account.

\section{Serology test}

The FMDV-specific antibody titers in immunized cattle were determined by a commercial liquid-phase-block ELISA (LPB-ELISA) kit (Lanzhou Veterinary Research Institute, China). Virus neutralization test (VNT) was performed according to the protocol described by the OIE. Serum samples were inactivated at $56{ }^{\circ} \mathrm{C}$ for $30 \mathrm{~min}$ and subjected to a microtiter neutralization assay on BHK-21 cells. Two-fold serially diluted sera were incubated with $100 \mathrm{TCID}_{50}$ of FMDV strain Asial/JSL/ GSZY/06 at $37{ }^{\circ} \mathrm{C}$ and $5 \% \mathrm{CO}_{2}$ for $1 \mathrm{~h}$, followed by infection of monolayers of BHK-21 cells in 96-well plates for $72 \mathrm{~h}$. Thereafter, the cells were examined for FMDVspecific cytopathic effect (CPE), and neutralization titers were calculated based on the Reed-Muench method.

\section{Statistical analysis}

All data were expressed as the mean value of five animals \pm SEM. Differences were considered statistically significant when $P<0.05$.

\section{Results}

Expression, purification, and characterization of FMDV VLPs VP0, VP1 and VP3, the capsid proteins of FMDV, have the ability to form empty capsids resembling the capsids of virions and share the same antigenicity as virions. To obtain the FMDV VLPs, the recombinant plasmid pETTRI-Asia1-VP310 containing the full-length of VP0, VP1 and VP3 genes in tandem was constructed. The recombinant expression vector was then transformed into BL21/DE3 competent cells and protein expression was induced by IPTG. SDS-PAGE results showed that the recombinant proteins of expected size were expressed (Fig. 1). As shown in Fig. 1a, the recombinant proteins were found in the supernatant of cells after disruption by homogenizer (Avestin), which is about $20 \%$ of the total soluble proteins. Western-blot results showed that the recombinant proteins reacted with FMDV positive serum (Fig. 1b). The soluble fractions from bioreactor massive production were purified by affinity chromatography with $\mathrm{Ni}^{2+}$ resins (Novagen, USA) to capture the His $\times 6$-fusion proteins. After washing with washing buffer (50 mM Tris- $\mathrm{HCl}, 0.3 \mathrm{M} \mathrm{NaCl}, 0.2 \mathrm{mM}$ EGTA, and $40 \mathrm{mM}$ imidazole), the target proteins were eluted by $500 \mathrm{mM}$ imidazole and treated with SUMO protease Ulp1 overnight at $4{ }^{\circ} \mathrm{C}$ to remove the SUMO tag. The digestion products were further purified by Q Sepharose Fast Flow anion exchange chromatographic purification. The total purification yield is around $20 \sim 35 \%$ (Table 1). SDS-PAGE showed that the purity of the final recombinant capsid protein reached $\sim 90 \%$ calculated by Quantity One (Bio-Rad) (Fig. 1c). The purified recombinant capsid proteins (VP0, VP1 and VP3) were assembled into VLPs in vitro. Dynamic light Scattering (DLS) results showed that almost $100 \%$ of purified capsid proteins form uniform high molecular weight complex, suggesting VLP formation (Fig. 2a). The assembled VLPs were diluted in $50 \mathrm{mM}$ Tris- $\mathrm{HCl}$ and submitted to TEM analysis, which showed that the in vitro assembled FMDV VLPs were rendered as hollow shapes with the diameters of $\sim 30 \mathrm{~nm}$ with 42000 fold magnification which consistent with the DLS results (Fig. 2b).

\section{Immune-reactivity in cattle induced by vaccination with VLP vaccine}

Cattles were divided into two groups (groups A and B) and submitted to vaccination with $200 \mu \mathrm{g}$ and $100 \mu \mathrm{g}$ FMDV VLP per cattle, respectively. The specific antiFMDV antibody responses were evaluated by commercial LPB-ELISA kits. As shown in Fig. 3, the specific antibodies were detected at $21 \mathrm{dpv}$ in all vaccinated animals, and the antibody levels decreased gradually thereafter (Fig. 3). The persistent periods (mean titer $\geq 128$ ) of FMDV-specific LPB antibodies in groups A and B were 6 and 3 months, respectively.

To further investigate the correlations between the dose and the persistent period of FMDV-specific antibodies, three more groups of cattle were inoculated with one dose $(200 \mu \mathrm{g}), 1 / 3$ dose, and 1/9 dose of FMDV VLP vaccine, respectively. FMDV-special ELISA and neutralization antibody titers were determined at 0,7 , 14, 21, and $28 \mathrm{dpv}$ for each cattle. As shown in Fig. 4, all 5 animals vaccinated with 1 dose and most of the 10 cattle vaccinated with $1 / 3$ dose and 1/9 dose FMDV VLP vaccine developed a detectable level FMDV ELISA antibody response at $7 \mathrm{dpv}$ with the titer reaching up of 1440 (Fig. 4). Notably, the antibody titers were always maintained at high levels for the whole experimental period from $7 \mathrm{dpv}$ till $28 \mathrm{dpv}$. Consistently, FMDVspecific neutralizing antibodies were detected only in few serum samples at $7 \mathrm{dpv}$, which increased gradually to reach the highest level at challenge (28 dpv) (Fig. 5). In contrast, the antibody titers in the control group were negative for the both LPBE and VNT groups.

\section{$\mathrm{PD}_{50}$ test using Asia1/JSL/GSZY/06}

The $\mathrm{PD}_{50}$ test was performed to assess the subunit vaccine potency by following the bovine potency test protocol described by the OIE to test the traditional inactivated FMDV vaccines. When challenged with FMDV strain Asial/JSL/GSZY/06, both the control animals exhibited typical FMD lesions on at least three feet by 5 day post-challenge ( $\mathrm{dpc}$ ), while vaccinated animals showed different levels of protection. All five cattle were 

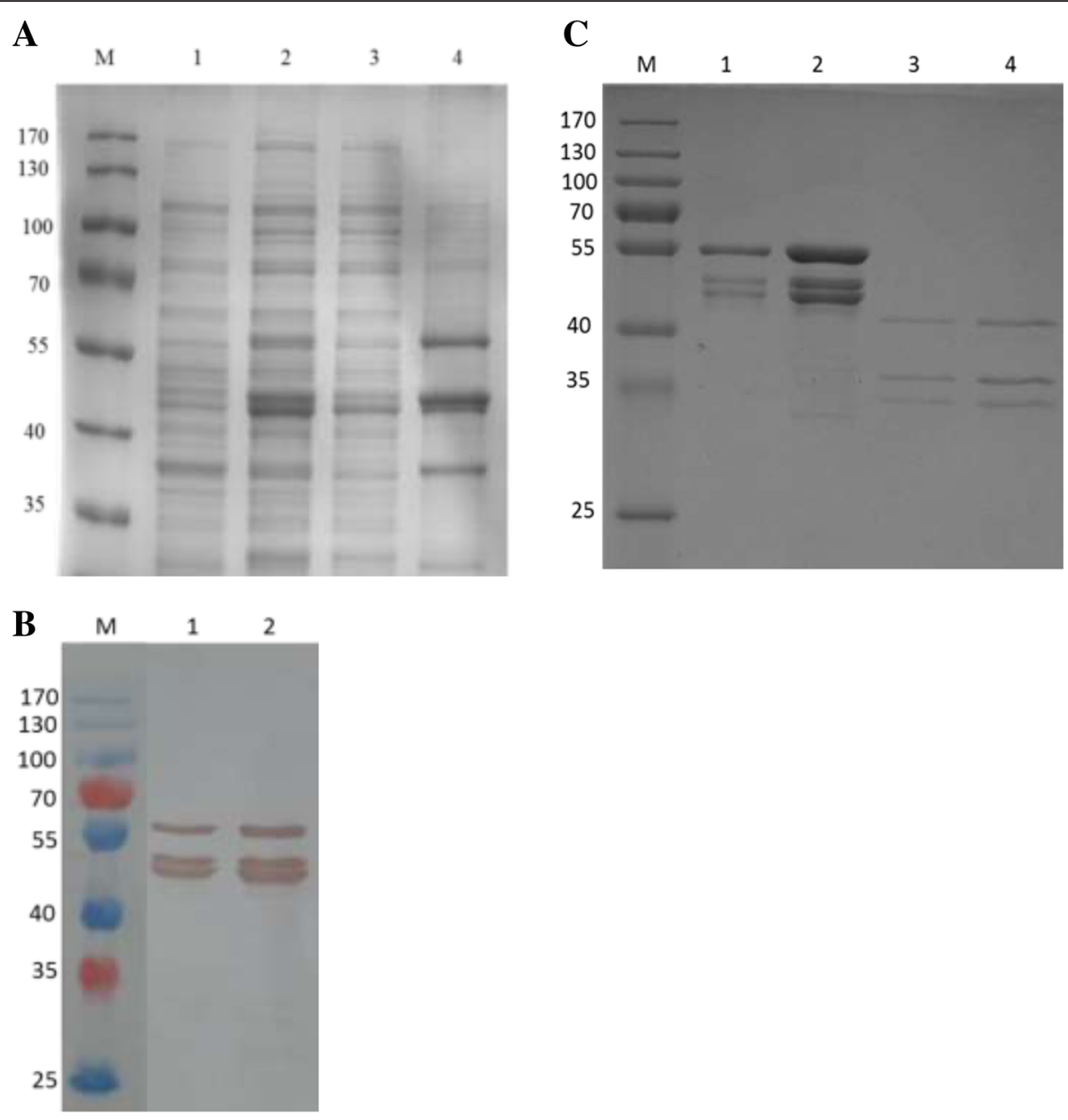

Fig. 1 SDS-PAGE and western-blot analysis of FMDV capsid proteins expressed in E. coli. a SDS-PAGE of FMDV capsid protein expression without purification. M, protein marker; lane 1, total protein without IPTG induction; lane 2, total protein after IPTG induction; lane 3, supernatant protein after IPTG induction; lane 4, pellet protein after IPTG induction; b Western-blot of FMDV capsid protein expression without purification. M, protein marker; lane 1, supernatant protein after IPTG induction; lane 2, pellet protein after IPTG induction; c M, protein marker; lane 1, purified protein complex (1 ul load); lane 2, purified protein complex (10ul load); lane 3, purified protein complexes after Ulp 1 protease treatment (1 ul load); lane 4, purified protein complexes after Ulp 1 protease treatment (10ul load)

considered completely protected in the 1 dose immunized group, three of the four cattle were protected in the $1 / 3$ dose group as one cattle in this group died after challenge with no typical lesions of FMD, and four of the five cattle were protected in the 1/9 dose group. The lesions in the $1 / 3$ dose and $1 / 9$ dose groups were

Table 1 Protein yield at different purification steps

\begin{tabular}{lll}
\hline Steps & Procedure & Protein recovery (\%) \\
\hline 1 & $\begin{array}{l}\text { Ni2+ affinity chromatography to capture } \\
\text { SUMO-VP proteins }\end{array}$ & $70 \%-80 \%$ \\
2 & $\begin{array}{l}\text { Cleavage of SUMO tag using Ulp1 to } \\
\text { release the un-tagged VP proteins }\end{array}$ & $60 \%-70 \%$ \\
3 & $\begin{array}{l}\text { Q Sepharose Fast Flow anion exchange } \\
\text { chromatography to obtain the pure VP } \\
\text { proteins }\end{array}$ & $50 \%-60 \%$ \\
& Total yield: & $20 \%-35 \%$ \\
\hline
\end{tabular}

detectable by $6 \mathrm{dpc}$ and the lesions were less severe compared to control group with lesion on only one foot. In this research, the result showed the vaccine potency of the batch immunized with the expressed antigens reached $11.75 \mathrm{PD}_{50}$ per dose (Table 2). These data indicated that the VLPs produced in E. coli elicited a protective immune response in cattle.

\section{Discussion}

FMD, one of the most devastating diseases of livestock, can cause significant economic losses worldwide, and remains the most important constraint to international trade in live animals and animal products. FMDV is divided into seven serotypes with no cross-protection conferred among the serotypes, and three of which exist in China, including serotypes O, Asia1, and A. FMDV serotype Asia1, an unique serotype of Asia, was first detected 


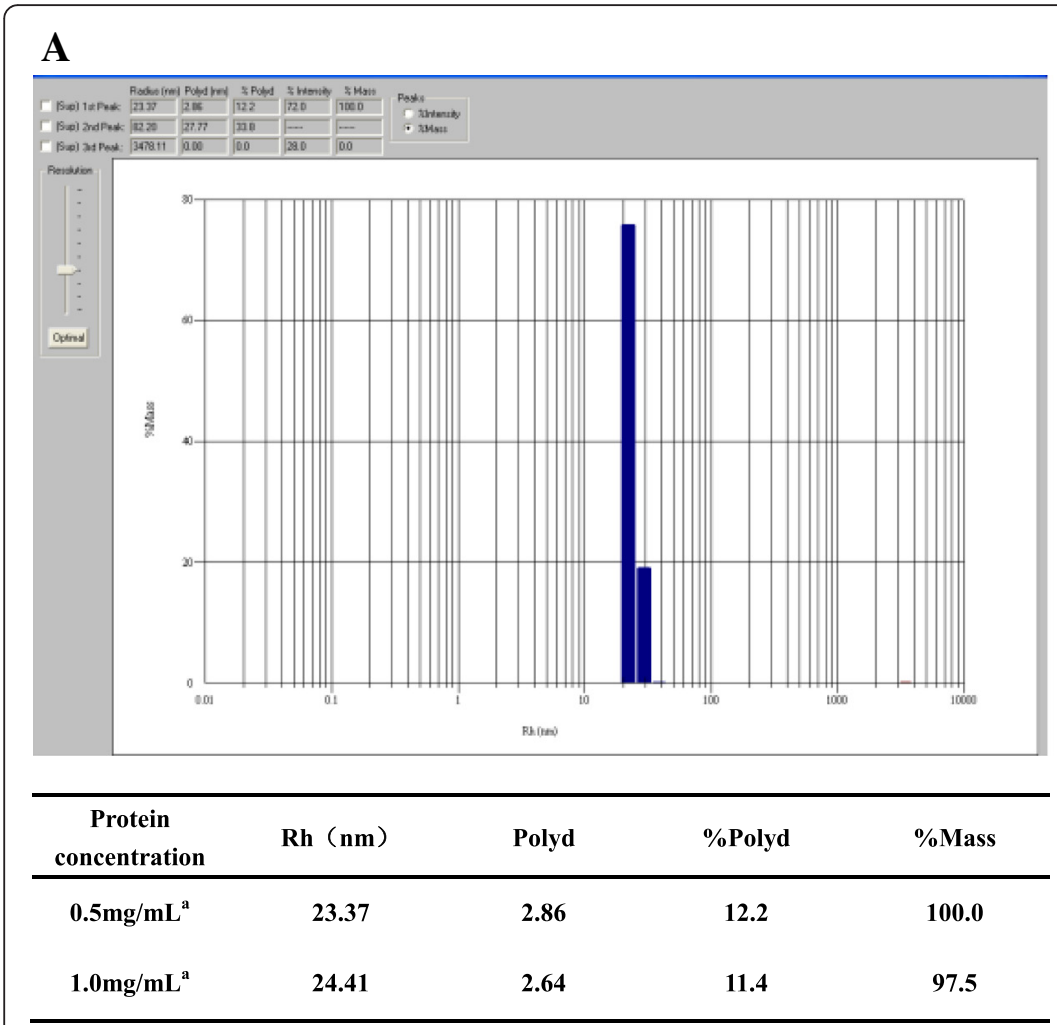

B

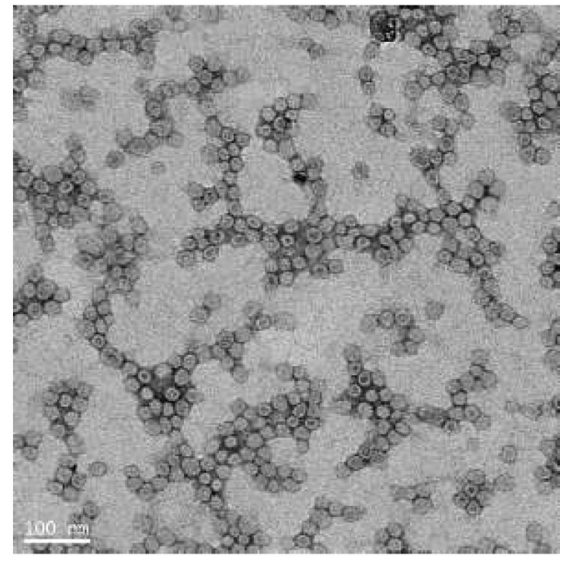

Note: ${ }^{\mathrm{a}}$ There was no significant difference between $0.5 \mathrm{mg} / \mathrm{mL}$ and $1.0 \mathrm{mg} / \mathrm{mL}$.

Fig. 2 Characterization of in vitro re-assembled FMDV VLPS. a DLS profile. The difference of DSL profile between two protein concentrations was determined by using t-test in GraphPad 5.0. Differences were considered statistically significant when $\mathrm{P}<0.05$. b Images of VLPs under electron microscope with $42000 \times$ magnification. The scale bar indicates $100 \mathrm{~nm}$

in samples collected in India in 1951 through 1952 and Pakistan in 1954 [15]. In China, serotype Asial became an important prevalent serotype after an outbreak recorded in Hong Kong in 2005 [16]. FMD vaccines are applied for prophylactic use in endemic areas worldwide, two vaccine types including

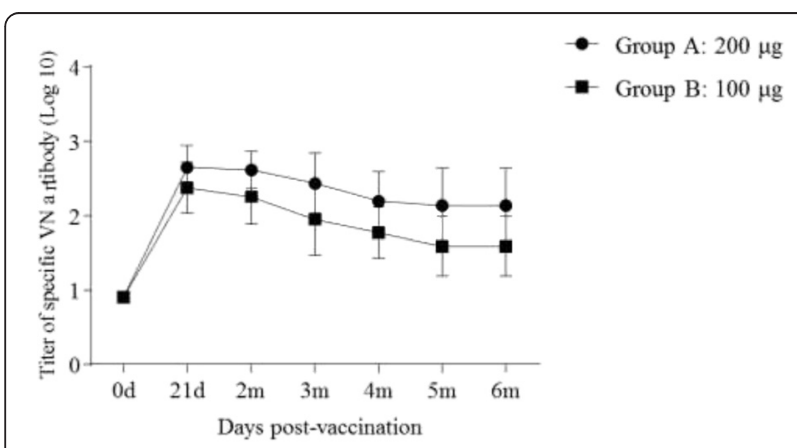

Fig. 3 FMDV-specific immune responses in cattle measured by LPBELISA. Groups of cattle $(n=5)$ immunized with 200ug or 100ug of FMDV VLPs. Blood samples were collected at 21 days postvaccination and 2, 3,4,5 and 6 months after vaccination for the LPBELISA detection. LPB-ELISA value $\geq 128$ ( $\log _{10} 2.1$ ) indicates above $99 \%$ protection to the viral challenge according to the manufacture's instruction traditional killed vaccines and synthetic peptide vaccines are commercially available and widely used in China.

Although the traditional inactivated vaccine has been proved effective, the development of a novel subunit FMDV vaccine that is safer, more effective and more

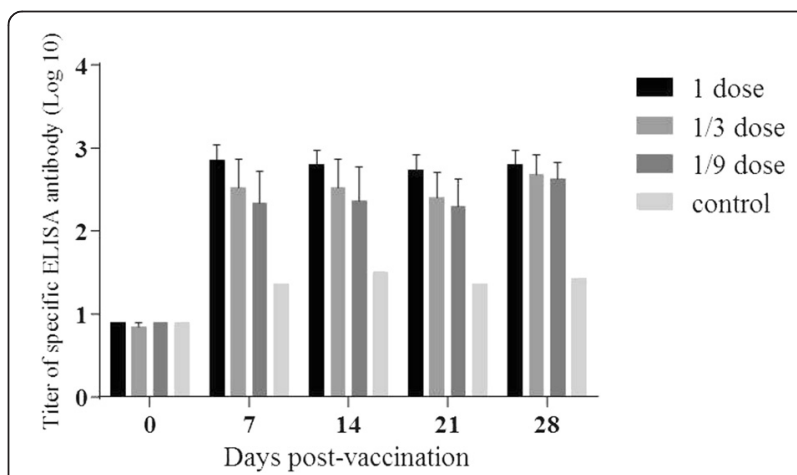

Fig. 4 FMDV-specific immune responses in cattle measured by LPB-ELISA in $\mathrm{PD}_{50}$ test. Blood samples were collected at 0, 7, 14, 21, and 28 days post-vaccination for the LPB-ELISA detection. LPB-ELISA value $\geq 128\left(\log _{10} 2.1\right)$ indicates above $99 \%$ protection to the viral challenge according to the manufacture's instruction. Each data points represents the mean \pm standard error of measurements for five cattle from immunized groups and for 2 cattle from control group 


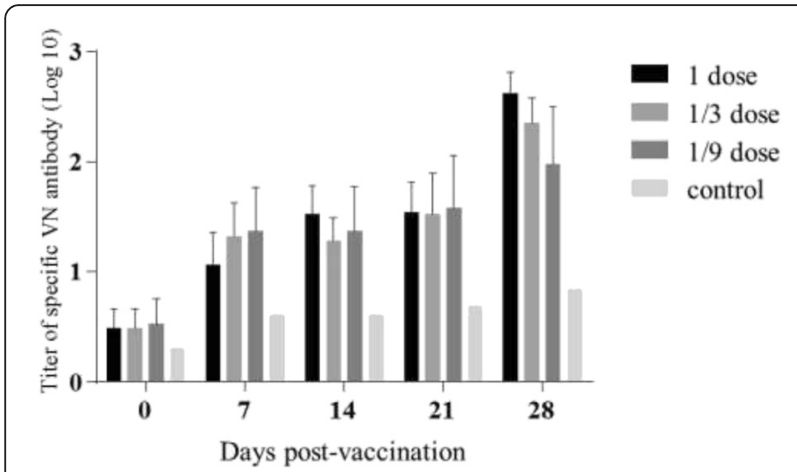

Fig. 5 FMDV-specific immune responses in cattle measured by virus neutralizing antibody test. Blood samples were collected at 0, 7, 14, 21 , and 28 days post-vaccination for the virus neutralizing antibody test. The virus neutralizing antibody was expressed by the average titers of cattle in one whole group at different time points. Each data points represents the mean \pm standard error of measurements for five cattle from immunized groups and for 2 cattle from control group

economical than traditional vaccines is essential to control FMDV spreading worldwide. Notably, FMDV is a non-enveloped RNA virus and each FMDV capsid subunit is formed by the assembly of a single copy of the structural proteins VP0, VP1, and VP3 coded by the P1 region of the viral genome. Among them, VP0 is digested to make capsid proteins VP2 and VP4 during virus maturation [17]. In previous reports, the fusion proteins of three FMDV capsid proteins, VP0, VP1 and VP3, have been successfully expressed in E. coli as SUMO-fusion format at $\sim 5 \mathrm{mg} / \mathrm{L}$ level by cotransformation of 2 or 3 plasmids with multiple antibiotic selections $[8,14]$. No robust protocols for large scale production and purification of FMDV VLPs were provided. In this study, we first made a single plasmid by constructing RBS-SUMO-VP3, RBS-SUMO-VP1 and RBS-SUMO-VP0 genes in tandem and driven by a single promoter with its own ribosome binding site, which led to the co-expression of SUMO-tagged VP3, VP1 and VP0 in E. coli, selected by only one single antibiotics. Compared with the previous reported production of FMDV vaccines, our protocol has the following advantages. 1) The genes encoding SUMO-tagged VP3, VP1 and VPO were constructed in tandem into a single plasmid and selected by a single antibiotics; 2) The SUMOtagged VP3, VP1 and VP0 proteins in this protocol were expressed at soluble form with the expression level as high as $\sim 50 \mathrm{mg} / \mathrm{L}$ by fermentation, which was ten times higher than the previously reported protocols $[8,14] ; 3$ ) The addition of SUMO tags not only facilitates the ensuing purification processes but preventing the aggregation of FMDV capsid proteins; 4) A robust purification protocol was provided to make well-assembled FMDV VLPs with the purify reaching $\sim 90 \%$ without using the

Table 2 Results of $\mathrm{PD}_{50}$ test. $\mathrm{PD}_{50}$ test was performed as described by the OIE. The bovine $\mathrm{PD}_{50}$ content of the vaccine was calculated based on the Reed-Muench method from each animal protected in each group

\begin{tabular}{|c|c|c|c|c|c|c|c|}
\hline \multirow[t]{2}{*}{ Groups } & \multirow{2}{*}{$\begin{array}{l}\text { Animal } \\
\text { No. }\end{array}$} & \multicolumn{2}{|c|}{ Antibody titer at $28 \mathrm{dpv}$} & \multirow{2}{*}{$\begin{array}{l}\text { Protection } \\
\text { Yes }\end{array}$} & \multirow{2}{*}{$\begin{array}{l}\text { Days of onset of lesion } \\
\text { - }\end{array}$} & \multirow{2}{*}{$\begin{array}{l}\text { Rate of protection } \\
5 / 5\end{array}$} & \multirow[t]{2}{*}{$\mathrm{PD}_{50}$} \\
\hline & & LPBE & VN & & & & \\
\hline & & LPBE & VN & Yes & - & $5 / 5$ & 11.75 \\
\hline \multirow[t]{5}{*}{1 dose } & 1558 & 360 & 64 & Yes & - & & \\
\hline & 1559 & 1440 & 64 & Yes & - & & \\
\hline & 1584 & 1440 & 724 & Yes & - & & \\
\hline & 1601 & 180 & 362 & Yes & - & & \\
\hline & 1602 & 720 & 512 & Yes & - & & \\
\hline \multirow[t]{5}{*}{$1 / 3$ dose } & 1534 & 1440 & 724 & Yes & - & $3 / 4$ & \\
\hline & 1548 & 1440 & 724 & No & D10 & & \\
\hline & 1562 & 90 & 724 & Death $^{a}$ & - & & \\
\hline & 1567 & 720 & 431 & Yes & - & & \\
\hline & 1576 & 180 & 76 & Yes & - & & \\
\hline \multirow[t]{5}{*}{ 1/9 dose } & 29 & 1440 & 724 & Yes & - & $4 / 5$ & \\
\hline & 77 & 360 & 54 & Yes & - & & \\
\hline & 1136 & 2880 & 724 & Yes & - & & \\
\hline & 1139 & 180 & 4 & No & D6 & & \\
\hline & 1580 & 360 & 512 & Yes & - & & \\
\hline \multirow[t]{2}{*}{ Control } & 1561 & 22 & 16 & No & D5 & $0 / 2$ & \\
\hline & 1590 & 45 & 8 & No & D5 & & \\
\hline
\end{tabular}

${ }^{a}$ One animal in this group died after challenge, but with no typical lesions of FMD 
industrial non-preferred size exclusion chromatographic purification or sucrose gradient ultracentrifugation steps, which dramatically reduced the cost of vaccine production. Moreover, the application of our in vitro assembled FMDV VLP vaccine has successfully elicited robust high level of $\mathrm{VN}$ antibodies in the tested cattle right after 28 dpv. Furthermore, high level of $\mathrm{VN}$ antibodies were detected in all cattle of 1 dose group and 1/3 dose group, and four out of five in $1 / 9$ dose group (Table 1). Unexpected, animal No. 1548 in 1/3 dose group displaying a high VNA titer showed no resistance to challenge. However, similar observation was also seen in previous studies of FMDV VLP subunit vaccine development $[6,8]$. Although the good correlation between VNA titer and protection to FMDV challenge was generally considered, the disparity sometimes was reported due to variation of individual cattle in resistance to the virus infection as well as other immunological parameters such as cellular immunity.

Surprisingly, in the $\mathrm{PD}_{50}$ test, the percentage protection values showed a dose independent manner since either 1 dose, 1/3 dose or 1/9 dose FMDV VLP subunit vaccine displays similar significant protection. This observation is also consistent with the earlier reports in the FMDV potency tests $[18,19]$. As reported by Goris et al. [18], the largest variation in the number of protected animals was observed in the lowest vaccine volume dose group, where the entire possible range of protected animals varied widely (from 0 to 5 ). Nevertheless, our $E$. coli-based FMDV VLP vaccines produced by large-scale fermentation have shown the great promise in protecting cattle from FMDV infection.

Notably, according to the World Organization for Animal Health (OIE) manual (OIE, 2012), the FMDV vaccine should contain at least $3 \mathrm{PD}_{50}$ and $6 \mathrm{PD}_{50}$ per dose for cattle when employed for routine prophylactic vaccination and emergency vaccination, respectively. In China, Ministry of Agriculture of China elevated the potency criteria of all commercial FMDV vaccines from 3 to $6 \mathrm{PD}_{50}$ per dose in 2013 (http://www.moa.gov.cn/zwllm/tzgg/tfw/201306/t20130 613_3491208.htm). Such standard demands the development and production of FMDV vaccines with potent protection. Strikingly, our E. coli-based FMDV VLP vaccines by fermentation achieve $11.75 \mathrm{PD}_{50}$ per dose in cattle. Hence, our approach in large scale production of FMDV VLP subunit vaccine by $E$. coli expression system should have direct impact in controlling FMD spreading worldwide.

\section{Conclusions}

In this study, the FMDV capsid proteins VP0, VP1, and VP3 were expressed in E. coli and assembled into VLPs in vitro in large scale by fermentation at $10 \mathrm{~L}$ scale.
VLP-based FMDV vaccine induced strong and persistent humoral immunity with the period of 6 months which indicated this vaccine candidate could function well against FMDV Asia1 infection.

\section{Abbreviations \\ DLS, dynamic light scattering; E.coli, Escherichia coli; FMDV, foot-and-mouth disease virus; IPTG, isopropyl- $\beta$-d-thiogalactoside; $\mathrm{PD}_{50}, 50 \%$ bovine protect- ive dose; SDS-PAGE, sodium dodecyl sulfate polyacrylamide gel electrophor- esis; VLP, virus-like particle}

\begin{abstract}
Acknowledgments
We would like to acknowledge the Cryo-Electron Microscopy Facility at CBIS/ NUS for the scientific and technical assistance. This work was supported by grant from Major Science and Technology Program in Henan Province (Grant No.131100110200 to K.T.) and Talent Entrepreneurship Awards from SIP, Suzhou city and Jiangsu Province, respectively (to Y.A.Y).

\section{Funding}

This work was supported by grant from Major Science and Technology Program in Henan Province (Grant No.131100110200 to K.T.) and Talent Entrepreneurship Awards from SIP, Suzhou city and Jiangsu Province, respectively (to Y.A.Y).
\end{abstract}

\section{Availability of data and materials}

The datasets supporting the conclusions of this article are included within the article.

\section{Authors' contributions}

$\mathrm{H}-\mathrm{YC}$, constructed the plasmids. $\mathrm{H}-\mathrm{YC}, \mathrm{BY}$, and $\mathrm{XM}$ purified and characterized FMDV VLPs. YX, YW, CL, HY, YX performed the animal trials. XH and Wengiang Pang performed the bacterial fermentation. YAY, $X L$ and $K T$ conceived of the study, drafted and finalized the manuscript. All authors read, edited and approved the final version of the manuscript.

\section{Competing interests}

The authors declare that they have no competing interests.

\section{Consent for publication}

Not applicable.

\section{Ethics approval and consent to participate}

The animal trial in this study was approved by the Animal Care and Ethics Committee of China National Research Center for Veterinary Medicine with reference number 2015062503, and conventional animal welfare regulations and standards were taken into account.

\section{Author details}

${ }^{1}$ National Research Center for Veterinary Medicine, Road Cuiwei, High-Tech District, Luoyang 471003, People's Republic of China. ${ }^{2}$ College of Animal Science and Veterinary Medicine, Henan Agricultural University, Zhengzhou, Henan 450002, People's Republic of China. ${ }^{3}$ National University of Singapore (Suzhou) Research Institute, 377 Lin Quan Street, Suzhou Industrial Park, Jiangsu 215123, China. ${ }^{4}$ Department of Biological Sciences and Centre for Bioimaging Sciences, National University of Singapore, 14 Science Drive 4, Singapore 117543, Singapore.

Received: 21 January 2016 Accepted: 21 June 2016

Published online: 02 July 2016

\section{References}

1. Perry BD, Rich KM. Poverty impacts of foot-and-mouth disease and the poverty reduction implications of its control. Vet Rec. 2007;160(7):238-41.

2. Barnett PV, Cox SJ, Aggarwal N, Gerber H, McCullough KC. Further studies on the early protective responses of pigs following immunisation with high potency foot and mouth disease vaccine. Vaccine. 2002;20(25-26):3197-208.

3. Grubman MJ, Baxt B. Foot-and-mouth disease. Clin Microbiol Rev. 2004; 17(2):465-93.

4. Rodriguez LL, Grubman MJ. Foot and mouth disease virus vaccines. Vaccine. 2009;27 Suppl 4:D90-94. 
5. Doel TR. FMD vaccines. Virus Res. 2003;91(1):81-99.

6. Zhang Z, Pan L, Ding Y, Zhou P, Lv J, Chen H, Fang Y, Liu X, Chang H, Zhang J, et al. Efficacy of synthetic peptide candidate vaccines against serotype-A foot-and-mouth disease virus in cattle. Appl Microbiol Biotechnol. 2015;99(3):1389-98.

7. Zhou G, Wang H, Wang F, Yu L. Recombinant adenovirus expressing type Asia1 foot-and-mouth disease virus capsid proteins induces protective immunity against homologous virus challenge in mice. Res Vet Sci. 2013; 94(3):796-802.

8. Guo HC, Sun SQ, Jin Y, Yang SL, Wei YQ, Sun DH, Yin SH, Ma JW, Liu ZX, Guo JH, et al. Foot-and-mouth disease virus-like particles produced by a SUMO fusion protein system in Escherichia coli induce potent protective immune responses in guinea pigs, swine and cattle. Vet Res. 2013:44:48.

9. Li Z, Yin X, Yi Y, Li X, Li B, Lan X, Zhang Z, Liu J. FMD subunit vaccine produced using a silkworm-baculovirus expression system: protective efficacy against two type Asia1 isolates in cattle. Vet Microbiol. 2011;149(1-2):99-103.

10. Li Z, Yi Y, Yin X, Zhang Z, Liu J. Expression of foot-and-mouth disease virus capsid proteins in silkworm-baculovirus expression system and its utilization as a subunit vaccine. PLoS One. 2008;3(5):e2273.

11. Ganges L, Borrego B, Fernandez-Pacheco P, Revilla C, Fernandez-Borges N, Dominguez J, Sobrino F, Rodriguez F. DNA immunization of pigs with footand-mouth disease virus minigenes: from partial protection to disease exacerbation. Virus Res. 2011;157(1):121-5.

12. Wang G, Pan $L$, Zhang $Y$, Wang $Y$, Zhang Z, Lu J, Zhou P, Fang Y, Jiang S. Intranasal delivery of cationic PLGA nano/microparticles-loaded FMDV DNA vaccine encoding IL-6 elicited protective immunity against FMDV challenge. PLoS One. 2011;6(11):e27605.

13. Balamurugan V, Renji R, Venkatesh G, Reddy GR, Nair SP, Ganesh K, Suryanarayana $\mathrm{W}$. Protective immune response against foot-and-mouth disease virus challenge in guinea pigs vaccinated with recombinant P1 polyprotein expressed in Pichia pastoris. Arch Virol. 2005;150(5):967-79.

14. Lee CD, Yan YP, Liang SM, Wang TF. Production of FMDV virus-like particles by a SUMO fusion protein approach in Escherichia coli. J Biomed Sci. 2009; 16:69.

15. Valarcher JF, Knowles NJ, Zakharov V, Scherbakov A, Zhang Z, Shang YJ, Liu ZX, Liu XT, Sanyal A, Hemadri D, et al. Multiple origins of foot-and-mouth disease virus serotype Asia 1 outbreaks, 2003-2007. Emerg Infect Dis. 2009; 15(7):1046-51.

16. Valarcher JF, Knowles NJ, Ferris NP, Paton DJ, Zakharov V, Sherbakov A, Shang YJ, Liu ZX, Liu X, Sanyal A, et al. Recent spread of FMD virus serotype Asia 1. Vet Rec. 2005;157(1):30.

17. Belsham GJ. Distinctive features of foot-and-mouth disease virus, a member of the picornavirus family; aspects of virus protein synthesis, protein processing and structure. Prog Biophys Mol Biol. 1993;60(3):241-60.

18. Goris N, Merkelbach-Peters P, Diev VI, Verloo D, Zakharov VM, Kraft HP, De Clercq K. European Pharmacopoeia foot-and-mouth disease vaccine potency testing in cattle: between test variability and its consequences. Vaccine. 2007;25(17):3373-9.

19. Mohana Subramanian B, Madhanmohan M, Sriraman R, Chandrasekhar Reddy RV, Yuvaraj S, Manikumar K, Rajalakshmi S, Nagendrakumar SB, Rana SK, Srinivasan VA. Development of foot-and-mouth disease virus (FMDV) serotype $\mathrm{O}$ virus-like-particles (VLPs) vaccine and evaluation of its potency. Antiviral Res. 2012;96(3):288-95.

\section{Submit your next manuscript to BioMed Central and we will help you at every step:}

- We accept pre-submission inquiries

- Our selector tool helps you to find the most relevant journal

- We provide round the clock customer support

- Convenient online submission

- Thorough peer review

- Inclusion in PubMed and all major indexing services

- Maximum visibility for your research

Submit your manuscript at www.biomedcentral.com/submit

C Biomed Central 